\title{
A novel splice mutation induces exon skipping of the EXT1 gene in patients with hereditary multiple exostoses
}

\author{
XIAOYAN GUO ${ }^{1}$, MINGRUI LIN ${ }^{2}$, WEI YAN $^{3}$, WENXU CHEN $^{1}$ and GUOLIN HONG ${ }^{4}$ \\ ${ }^{1}$ Department of Laboratory Medicine, Fuzhou Second Hospital, Fuzhou, Fujian 350007; \\ ${ }^{2}$ Intensive Care Unit, The Affiliated People's Hospital of Fujian Traditional Medical University, \\ Fuzhou, Fujian 350004; ${ }^{3}$ Department of Bone Tumors, Fuzhou Second Hospital, Fuzhou, Fujian 350007; \\ ${ }^{4}$ Department of Laboratory Medicine, The First Affiliated Hospital of Xiamen University, Xiamen, Fujian 361003, P.R. China
}

Received July 3, 2018; Accepted November 16, 2018

DOI: $10.3892 /$ ijo.2019.4688

\begin{abstract}
The molecular mechanism of hereditary multiple exostoses (HME) remains ambiguous and a limited number of studies have investigated the pathogenic mechanism of mutations in patients with HME. In the present study, a novel heterozygous splice mutation (c.1284+2del) in exostosin glycosyltransferase 1 (EXT1) gene was identified in a three-generation family with HME. Bioinformatics and TA clone-sequencing indicated that the splice site mutation would result in exon 4 skipping. Reverse transcription-quantitative polymerase chain reaction ( $\mathrm{RT}-\mathrm{qPCR}$ ) revealed that the expression levels of wild-type EXT1/EXT2 mRNA in patients with HME were significantly decreased, compared with normal control participants $(\mathrm{P}<0.05)$. Abnormal EXT1 transcript lacking exon 4 (EXT1-DEL) and full-length EXT1 mRNA (EXT1-FL) were overexpressed in 293-T cells and Cos-7 cells using lentivirus infection. RT-qPCR demonstrated that the expression level of EXT1-DEL was significantly increased, compared with EXT1-FL (17.032 vs. 6.309, respectively; $\mathrm{P}<0.05)$. The protein encoded by EXT1-DEL was detected by western blot analysis, and the level was increased, compared with EXT1-FL protein expression. Immunofluorescence indicated that the protein encoded by EXT1-DEL was located
\end{abstract}

Correspondence to: Professor Guolin Hong, Department of Laboratory Medicine, The First Affiliated Hospital of Xiamen University, 55 Zhenghai Road, Xiamen, Fujian 361003, P.R. China

E-mail: xmhg19899@sina.com

Mr. Wenxu Chen, Department of Laboratory Medicine, Fuzhou Second Hospital, 47 Shangteng Road, Fuzhou, Fujian 350007, P.R. China

E-mail: chenwx1969@126.com

Abbreviations: HME, hereditary multiple exostoses; HS, heparan sulfate; NMD, nonsense-mediated mRNA decay; HSF, Human Splicing Finder; BDGP, Berkeley Drosophila Genome Project

Key words: hereditary multiple exostoses, exostosin glycosyltransferase 1 gene, splice mutation, lentivirus infection in the cytoplasm of Cos-7 cells, which was consistent with the localization of the EXT1-FL protein. In conclusion, the present study identified a novel splice mutation that causes exon 4 skipping during mRNA splicing and causes reduced expression of EXT1/EXT2. The mutation in EXT1-DEL generated a unique peptide that is located in the cytoplasm in vitro, and it expands the mutation spectrum and provides molecular genetic evidence for a novel pathogenic mechanism of HME.

\section{Introduction}

Hereditary multiple exostoses (HME), also termed hereditary multiple osteochondroma, is an autosomal dominant inherited disease characterized by the development of multiple exostoses, predominantly located on the limbs, shoulder blades, ribs, and pelvis (1). Osteochondromas are frequently adjacent to the growth plates of bones, and can increase in size and number until growth plates close as the child stops developing. The exostoses can result in numerous health problems, including skeletal bowing and deformities, growth restriction, and nerve and blood vessel compression (1,2). As a common benign bone tumor, HME is estimated to occur at a rate of 1/50,000 cases; however, HME progresses into chondrosarcomas or osteosarcomas in $\sim 2 \%$ of patients (3-6).

Heterozygous germline mutations in the exostosin glycosyltransferase 1 (EXT1) and EXT2 genes are exhibited in $>90 \%$ of HME cases (7-9). Although no EXT1 or EXT2 germline mutations are detected in certain cases, somatic mosaic mutations were identified in one HME case (10). In patients with HME, $10 \%$ of mutations are spontaneous and $90 \%$ of affected individuals have a family history of HME (11). Additionally, $80 \%$ of mutations in patients with HME are truncation mutations, including nonsense, frameshift and splice site mutations, which commonly introduce premature stop codons during translation, or result in partial or entire loss of gene function (9). EXT1 and EXT2 are tumor suppressor genes that encode glycosyltransferases $(7,12)$, EXT1 and EXT2 form a hetero-oligomeric complex in the Golgi body that catalyzes chain elongation during the biosynthesis of heparan sulfate (HS) (13). HS has a key role in chondrocyte proliferation and endochondral ossification (14). Therefore, 
heterozygous mutations in the EXT1 or EXT2 gene theoretically result in a reduction in systemic HS levels by $\sim 50 \%$ in HME individuals. However, it has been reported that haploinsufficiency may not always result in osteochondroma formation. When HS levels are significantly decreased, but not lost completely, a second event, such as loss-of-heterozygosity or compound heterozygous mutations, appears to be the major cause of HME development, which has been confirmed in animal models and also reported in a number of patients with HME (15-19).

Recently, 436 mutations in EXT1 and 223 mutations in EXT2 have been reported in the Multiple Osteochondroma Mutation Database (http://medgen.ua.ac.be/LOVDv.2.0/home. php), including various splicing mutations (11). Alternative splicing is ubiquitous in mammals, and is a major contributor to molecular diversity and complexity, and gene regulation; additionally, alternative splicing is required for numerous critical biological processes in development and disease, including regulation of cell growth, hormone responsiveness and cancer $(20,21)$. However, once mutations exist in splicing elements or splicing signal sequences, particularly at $3^{\prime}$ and 5 ' splice sites, normal splicing of mRNA and translation will be disrupted, which can cause exon skipping or aberrant splicing, where new splicing sites are created, resulting in truncated proteins with potentially reduced expression and function (21). For example, dysregulation of alternative splicing has been demonstrated to be associated with various human diseases, including cancer, muscular dystrophies, neurodegenerative diseases and obesity (21).

A number of splicing mutations have been detected in patients with HME, and the molecular mechanisms are reported to involve the creation of new splice sites or exon skipping due to splicing mutations, resulting in early termination of translation and the degradation of truncated peptides via nonsense-mediated mRNA decay (NMD) $(22,23)$. In the present paper, a splice mutation in EXT1 (c.1284+2del) was identified in a three-generation Chinese family with HME. Skipping of EXT1 exon 4 was verified by TA cloning and sequencing of EXT1 mRNA from the patients with HME. No premature stop codon was produced by the skipping of exon 4; however, the expression levels of EXT1/EXT2 mRNA were notably reduced in the patients, as indicated by reverse transcription-quantitative polymerase chain reaction (RT-qPCR). In vitro, the truncated mutant protein was detected in the cytoplasm when expressed in Cos-7 cells. Thus, whether mutant EXT1 or EXT2 proteins are biologically functional requires further research, but the decrease in the expression of wild-type EXT1/EXT2 proteins will hinder the process of HS polymerization and chain elongation.

\section{Materials and methods}

Study participants, cell culture and reagents. Peripheral blood samples were collected from a Chinese family with HME in three generations from May 2013 to March 2015 (Fig. 1A). The HME diagnosis was produced according to their clinical manifestations and physical examinations, including $\mathrm{X}$-ray, computed tomography and pathological sections (24). Osteochondromas tissue was stained with hematoxylin and eosin for $5 \mathrm{~min}$ at room temperature. In the present study, two patients with HME (III 1, the proband, male, 31 years; II 2, the mother of the proband, female, 62 years), one normal family member (II 1, the father of the proband, male, 65 years) and one healthy individual (normal physical examination, male, 31 years) were enrolled in mutation analysis of EXT1 and EXT2 genes. The proband (III 1) was the first person who required surgical intervention in the family with $\mathrm{HME}$, and was an inpatient of the Department of Bone Tumors of Fuzhou Second Hospital (Fuzhou, China), the healthy individual was an inpatient at the Medical Examination Center of Fuzhou Second Hospital. The samples were collected together when the proband was in hospital for surgery. Written informed consent was obtained from all participants, and the study was approved by the Ethics Committee of Fuzhou Second Hospital [approval no. (2014) 63].

293-T and Cos-7 (originating from African green monkey kidney fibroblasts) cell lines (Cell Bank of the Chinese Academy of Sciences, Shanghai, China) were cultured in Dulbecco's modified Eagle's medium (DMEM) supplemented with $10 \%$ fetal bovine serum (both from Gibco; Thermo Fisher Scientific, Inc., Waltham, MA, USA), and incubated at $37^{\circ} \mathrm{C}$ incubator in an atmosphere containing $5 \% \mathrm{CO}_{2}$. Cells were passaged at $80 \%$ confluency by digestion with trypsin (Gibco; Thermo Fisher Scientific, Inc.).

Mutation screening for EXT1 and EXT2 genes. Genomic DNA of the participants was extracted from peripheral blood according to the procedures of the SE Blood DNA kit (Omega Bio-Tek, Inc., Norcross, GA, USA). DNA samples were used for mutation screening of the coding exons and the adjacent introns of EXT1 (GenBank NG_007455.2) (https://www.ncbi.nlm.nih.gov/nuccore/NG_007455.2) and EXT2 (GenBank NG_007560.1) (https://www.ncbi.nlm.nih. gov/nuccore/NG_007560.1) genes using previously reported primer sequences $(22,25)$. The products of the amplified sequences were observed on a $2 \%$ agarose gel and purified with HiBind ${ }^{\circledR}$ columns using an E.Z.N.A. ${ }^{\circledR}$ Cycle-Pure kit (Omega Bio-Tek, Inc.). Bidirectional sequencing was performed on purified products using an ABI 3730 XL genetic analyzer (Applied Biosystems; Thermo Fisher Scientific, Inc.). The possible pathogenic mutation screened from the sequencing would be proved to be a novel variant or a reported one in the ExAc database (http://exac.broadinstitute. org/gene/ENSG00000182197)

Bioinformatics analysis and prediction. Several web-based programs with different algorithms were used to analyze the potential effect of mutations on exon splicing. Mutation Taster and Protein Variation Effect Analyzer (PROVEAN) were selected for pathogenicity prediction. Mutation Taster uses a Bayes classifier to predict the disease potential of an alteration (mutationtaster.org/) and PROVEAN is a software tool that predicts whether an amino acid substitution or indel has an impact on the biological function of a protein (provean.jcvi. org/index.php). The CRYP-SKIP algorithm (http://cryp-skip. img.cas.cz/) uses multiple logistic regression to predict the two aberrant transcripts from the primary sequence, and was applied in the present study to estimate the probability of cryptic splice-site activation $(P)$ and exon skipping (1- $P$ ) due to a splicing mutation (26). The Berkeley Drosophila 
A

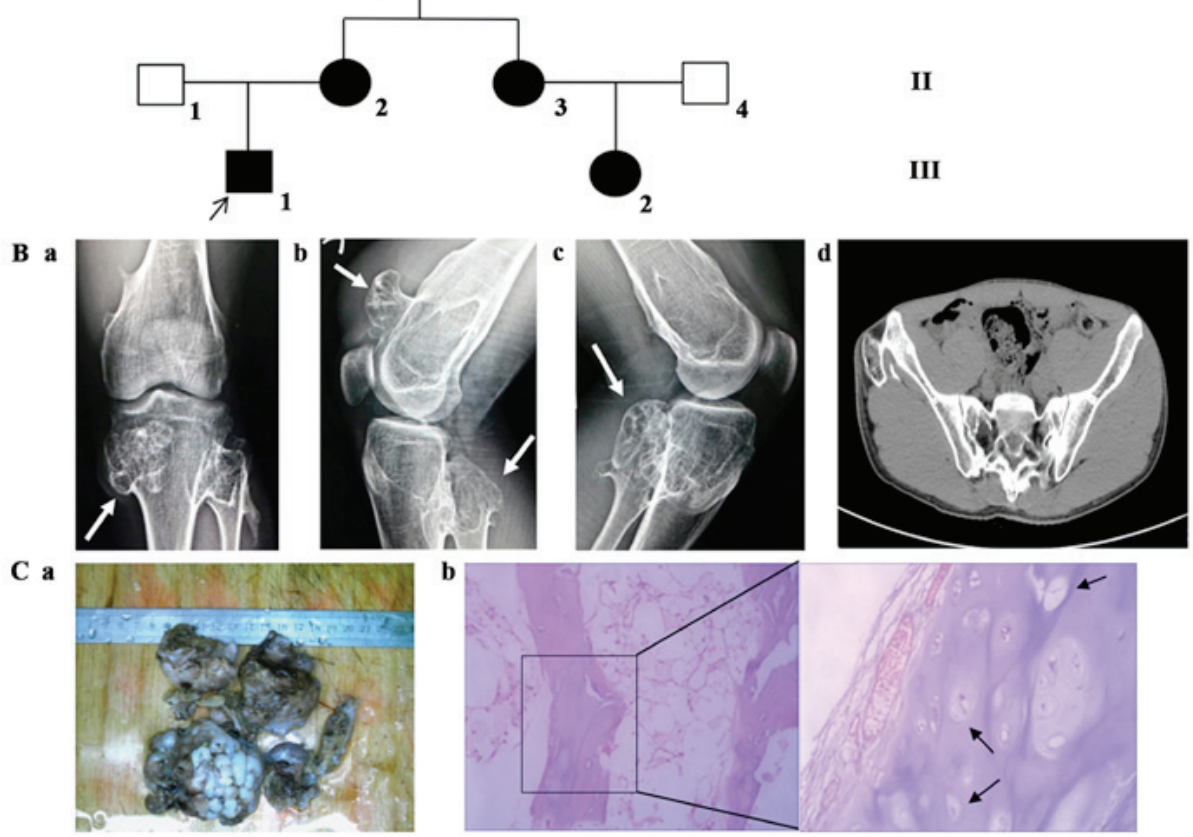

Figure 1. Pedigree and examinations of the proband. (A) Pedigree of the family with HME cases. The proband is indicated with a black arrow. (B-a) Osteochondroma were indicated with white arrows in the proximal end of the tibias, (B-b) proximal end of the fibulas, (B-c) distal end of the femurs and (B-d) the hipbones of the pelvis. (C) Pathological examination of the proband. (C-a) Tumors resected from the proband and (C-b) osteochondromas tissue stained with hematoxylin and eosin (C-a, x100 magnification; C-b, x400 magnification). Black arrows indicate osteochondromas in the third picture. HME, hereditary multiple exostoses.

Genome Project (BDGP) (http://www.fruitfly.org/about/index. $\mathrm{html}$ ) algorithm accurately distinguishes between donor and acceptor sites using a generalized hidden Markov model. As a splice site prediction program, it predicts cryptic splice sites and highlights changes in splice sites following input of a mutant sequence. The Human Splicing Finder (HSF) (http://www.umd.be/HSF3/) is an online tool that uses various algorithms to predict the effects of mutations on splicing signals or to identify splicing motifs in any human sequence. It has been previously used to predict the effects caused by splicing mutations (27).

Analysis of EXT1 and EXT2 mRNA. Total RNA was obtained from the venous blood of two patients with HME and normal controls according to the instructions of a QIAamp RNA Blood Mini kit (Qiagen GmbH, Hilden, Germany). Total RNA (5 $\mu \mathrm{g}$ ) was reverse transcribed into cDNA using random primers based of a PrimeScript ${ }^{\mathrm{TM}}$ 1st Strand cDNA Synthesis kit (Takara Biotechnology Co., Ltd., Dalian, China), and the synthesized cDNA was used as a template for PCR amplification of EXT1 with the following primers: 5 '-atgcaggccaaaaaacgctatt-3' (forward); and 5'-tcaaagtcgctcaatgtctcg-3' (reverse). LA Taq (Takara Biotechnology Co., Ltd.) was used as the DNA polymerase under the conditions: $94^{\circ} \mathrm{C}$ for $5 \mathrm{~min}, 94^{\circ} \mathrm{C}$ for $30 \mathrm{sec}$, annealing for $30 \mathrm{sec}\left(53^{\circ} \mathrm{C}\right), 72^{\circ} \mathrm{C}$ for $45 \mathrm{sec}$ for a total of 30 cycles; and the last cycle was extended at $72^{\circ} \mathrm{C}$ for $10 \mathrm{~min}$ (ABI 2720 Thermal Cycler; Applied Biosystems; Thermo Fisher Scientific, Inc.). The PCR products were separated by $1 \%$ agarose gel electrophoresis and visualized using ethidium bromide staining (Sangon Biotech Co., Ltd., Shanghai, China) for $\sim 1 \mathrm{~h}$ at room temperature. The products were purified using
HiBind $^{\circledR}$ columns and then cloned into the PGEM-T Easy vector (Promega Corporation, Madison, WI, USA). Ligation products were transformed into XL1-blue bacteria and cultured in ampicillin/X-gal/IPTG plates. Following transformation, $\sim 50$ positive clones were selected randomly from the proband, the mother and normal control, then cultured separately in a shaker overnight at $37^{\circ} \mathrm{C}$. Following extraction, the plasmids of the three groups were sequenced to search for the potential abnormal alternative transcripts in the individuals with HME, and the percentage of the mutated transcripts was evaluated with respect to the normal control.

cDNA from peripheral blood lymphocytes of the proband, the mother and normal control was amplified by two-step RT-qPCR according to the protocol of a SYBR ${ }^{\circledR}$ Ex Taq Reagent kit (Takara Biotechnology Co., Ltd.) using the LightCycler480 Real-Time PCR system (Roche Diagnostics, Indianapolis, IN, USA). qPCR was performed using the following primers: EXT1 (GenBank NM_000127.2), forward 5'-CATAGGCGATGAGAGATTGT-3', and reverse 5'-CAA GAATTGTGTCTGCTGTC-3' (fragment size, 97 bp); abnormally spliced EXT1, forward 5'-GTGATGCTCAGCAAT GGATG-3', and reverse 5'-TCTGTCCTGAATAATCTGTA-3' (fragment size, 108 bp); and EXT2 (GenBank NM_207122.1), forward 5'-GGCTACGATGTCAGCATTCCTG-3', and reverse 5'-GGCTTCTAGGTCCTCTCTGTAC-3' (fragment size, $141 \mathrm{bp}) .18 \mathrm{~s}$ rRNA was amplified simultaneously as an internal control (forward 5'-CGACGACCCATTCGAACGTCT-3', and reverse 5'-CTCTCCGGAATCGAACCCTGA-3'; fragment size, $102 \mathrm{bp}$ ). The conditions of RT-qPCR were as follows: $95^{\circ} \mathrm{C}$ for $3 \mathrm{~min}, 95^{\circ} \mathrm{C}$ for $15 \mathrm{sec}, 60^{\circ} \mathrm{C}$ annealing for $15 \mathrm{sec}$, $72^{\circ} \mathrm{C}$ for $20 \mathrm{sec}$ for a total of 39 cycles, $95^{\circ} \mathrm{C}$ for $5 \mathrm{sec}$, melt 
curve $60-95^{\circ} \mathrm{C}$ at increments for $0.5^{\circ} \mathrm{C}$ every $5 \mathrm{sec}\left(\mathrm{CFX} 96^{\mathrm{TM}}\right.$ Real-Time System; Bio-Rad Laboratories, Inc., Hercules, CA, USA). The $2^{-\Delta \Delta C q}$ method was used to calculate relative gene expression (28).

Lentiviral transduction in vitro and observation of growth condition. The full-length coding sequence of EXT1 gene (EXT1-FL) was amplified using cDNA from the peripheral blood of a normal participant, and the abnormal mutant transcript of EXT1 (EXT1-DEL) was amplified using the aforementioned recombinant plasmids from TA cloning and sequencing as the template, which matched the NCBI reference sequence (GenBank NM_000127.2), except for exon 4. The products of the amplified transcripts were confirmed by sequencing. GV358-EXT1-FL and GV358-EXT1-DEL lentiviral vectors were constructed, and were amplified and titrated based on the manufacturer's instructions (Shanghai GeneChem Co., Ltd., Shanghai, China) (29). A GV358-GFP vector was used as a negative control (NC). 293-T and Cos-7 cells were seeded in 6-well plates at a density of $5 \times 10^{5}$ cells $/ \mathrm{ml}$ of DMEM and then co-infected with GV358-EXT11-FL, GV358-EXT1-DEL and empty vector (GV358-GFP vector), which were mixed with Polybrene (Shanghai GeneChem Co., Ltd.) at a multiplicity of infection of 10. Cell infection efficiency and growth state were assessed by observation of green fluorescent protein (EXT1-GFP fusion protein) and cell morphological characteristics at $24 \mathrm{~h}$ after infection using a fluorescence microscope (x200 magnification; Olympus Corporation, Tokyo, Japan).

RNA extraction and RT-qPCR. Based on the TRIzol ${ }^{\circledR}$ (Shanghai Pufei Biotechnology Co., Ltd., Shanghai, China) method, total RNA was extracted from three groups of 293-T cells infected with lentivirus after $48 \mathrm{~h}$. Subsequently, RNA was reverse transcribed into cDNA using Promega M-MLV kits (Promega Corporation). GV358-EXT1-FL, GV358-EXT1-DEL and NC were simultaneously detected by two-step RT-qPCR under the same conditions aforementioned. The primer sequences used were as follows: Forward 5'-TTTTCTGCCCTACGACAA CAT-3', and reverse 5'-ACACTGTGAAGGCGAAATCCA-3', fragment size, $98 \mathrm{bp}$. As an internal control, GAPDH was also amplified using the following primers: Forward 5'-TGACTT CAACAGCGACACCCA-3' and reverse 5'-CACCCTGTT GCTGTAGCCAAA-3'; fragment size, 121 bp.

Protein extraction and western blotting. Total protein was isolated from cultured 293-T cells and Cos-7 cells at $72 \mathrm{~h}$ after infection. The protein concentration was measured using a Bicinchoninic Acid Protein Assay kit (Beyotime Institute of Biotechnology, Shanghai, China) and $30 \mu \mathrm{g}$ protein from each group was separated via SDS-PAGE on $10 \%$ gels, then blotted onto polyvinylidene difluoride membranes (cat. no. IPVH00010; EMD Millipore, Billerica, MA, USA). Following electrophoresis, membranes were blocked with 5\% bovine serum albumin (BSA; Thermo Fisher Scientific, Inc.) for $1 \mathrm{~h}$ at room temperature. Membranes were probed with mouse anti-Flag monoclonal antibody (cat. no. RLM3001; Suzhou Ruiying Biotechnology Co., Ltd., Suzhou, China; 1:3,000 dilution) and rabbit anti-human EXT1 (cat. no. ab126305; Abcam, Cambridge, UK; 1:3,000 dilution) to detect EXT1 and the novel truncated peptide, which targeted the 205-335 amino acid sequences of human EXT1, a region upstream of exon 4 (overnight at $4^{\circ} \mathrm{C}$ ). Peroxidase-conjugated goat anti-mouse IgG (cat. no. A0216) and goat anti-rabbit IgG (cat. no. A0208) secondary antibodies were incubated for $1 \mathrm{~h}$ at room temperature (both from Beyotime Institute of Biotechnology; both 1:10,000 dilution). GAPDH was also detected using mouse anti-GAPDH monoclonal antibody (cat. no. RLM3029; Suzhou Ruiying Biotechnology Co., Ltd.; 1:3,000 dilution) as an endogenous control for the western blot analysis under the same conditions aforementioned. Finally, the membranes were developed using enhanced chemiluminescence (ECL-Plus) reagent (Thermo Fisher Scientific, Inc.) and exposed to x-ray film (Carestream Health, Inc., Rochester, NY, USA).

Immunofluorescence analysis and subcellular localization detection using laser scanning confocalmicroscopy. Following infection for $48 \mathrm{~h}$, the monolayer of Cos- 7 cells was washed with PBS (3 times), fixed using 4\% paraformaldehyde under room temperature for $30 \mathrm{~min}$, permeabilized $(0.02 \%$ Triton X-100), blocked (5\% BSA) for $30 \mathrm{~min}$ at room temperature and then incubated at $4^{\circ} \mathrm{C}$ overnight with rabbit anti-human EXT1 (cat. no. 126305; Abcam; 1:200 dilution). Following being washed with PBS (3 times), cells were incubated with Alexa Fluor ${ }^{\circledR}$ 555-conjugated donkey anti-rabbit antibody (cat. no. A0453; Beyotime Institute of Biotechnology; 1:500 dilution) avoiding light for $2 \mathrm{~h}$ at room temperature. Finally, stained monolayer cells were washed with PBS three times and observed under a laser scanning confocal microscope (x630 magnification; Leica Microsystems GmbH, Wetzlar, Germany). The nucleus of the cells was stained with DAPI (cat. no. C1002; Beyotime Institute of Biotechnology).

Statistical analysis. $\chi^{2}$ test was used to evaluate the statistical significance of the result of TA cloning sequence (proportion). One-way analysis of variance with Least Significant Difference test was used to evaluate the statistical significance of the results of RT-qPCR, and they were expressed as the mean \pm standard error of the mean [SPSS 19.0 (IBM Corp., Armonk, NY, USA)]. $\mathrm{P}<0.05$ was considered to indicate a statistically significant difference.

\section{Results}

Clinical data of the family with HME. According to information provided by the proband, at least five individuals of the family were suspected to have multiple exostoses. However, only three members (the proband and his parents) participated in the present study and agreed to publication (Fig. 1A). The proband (III 1) had exhibited exostoses around the joints of the hips, knees, wrists, and ankles for $>20$ years. Furthermore, imaging (X-ray and computed tomography) and pathological sections confirmed the diagnosis (Fig. 1B and C). The mother had relatively minor symptoms according to the examination conducted.

Mutation screening and identification of a novel mutation $($ c.1284+2del) in EXT1. Sequencing results of the coding region and adjacent intronic sequences in EXT1/EXT2 genes of the proband revealed that there was a heterozygous deletion 


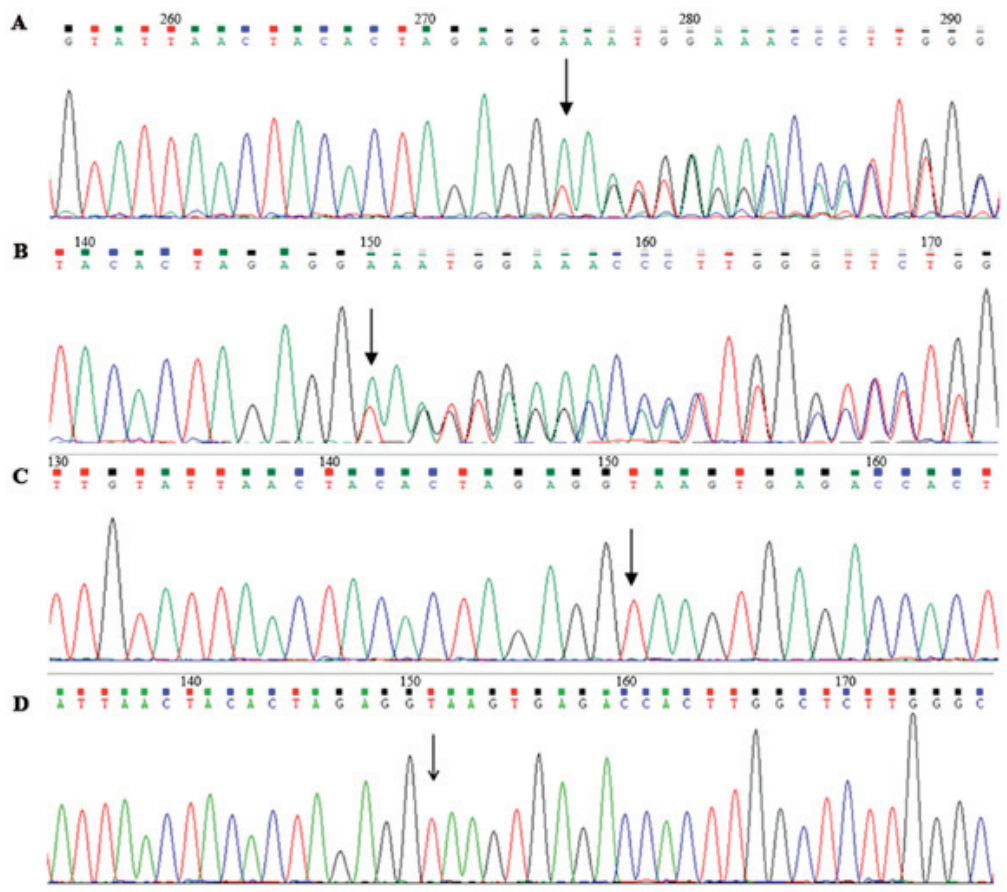

Figure 2. Sequencing results of exon 4 and adjacent intronic fragment of exostosin glycosyltransferase 1 gene. (A) Proband, (B) mother and (C) father from the family with hereditary multiple exostoses. (D) Normal control participants. The black arrows indicate the point of mutation.

(c.1284+2del) in intron 4 of the EXT1 gene, but no mutation was determined in the EXT2 gene (Fig. 2A). Furthermore, DNA sequencing identified the same alteration in the mother (II 2) (Fig. 2B); however, the mutation was not exhibited in the normal father or the healthy participants (Fig. 2C and D), and it was not reported in the ExAc database. The mutation spectrum indicated that the delete mutation was co-segregated with HME in this family. Sequence analysis indicated that c.1284+2del is an intron variation at the $5^{\prime}$ splice site (AGgt) of exon 4.

Abnormal splicing and exon skipping in EXT1 gene. Predictions from multiple bioinformatics databases revealed that the splicing mutation may cause two potential effects in the mRNA: Skipping of exon 4, or loss of the primary 5 splice site (AGGT) and activation of an adjacent cryptic splice site. The CRYP-AGgt analysis revealed that the probability of exon 4 skipping was 0.69 , whereas the probability of new cryptic splice site activation was 0.31 (Fig. 3A). BDGP predicted that the mutation caused the splice site to disappear at the mutational site. The HSF tool also indicated that AGgt was absent due to the mutation; however, it also suggested that a novel splice site (AAgt) emerged 3 bp downstream of AGgt. Mutation Taster predicted that c.1284+2del may disrupt normal splicing and that it was a disease-causing mutation that may affect the protein function. The novel polypeptide lacking amino acids from 389 to 428 of exostosin-1 protein (I389_E428del) created by exon 4 skipping was also indicated to be deleterious by PROVEAN analysis (data not shown).

TA cloning and sequencing of the targeted fragments of the two patients identified a notable number of abnormal transcripts with exon 4 skipping in EXT1 mRNA (proband 66.7\%, mother, $58.5 \%, \mathrm{P}<0.05$; Fig. 3B; Table I); however, no aberrantly spliced transcripts with cryptic splice site were identified in the patients.
Furthermore, although the ratio of transcripts with exon 4 jumping in EXT1 was increased in the proband, compared with the mother, there was no significant statistical difference between them $(\mathrm{P}>0.05$; Table I). The results from TA cloning and sequencing were almost consistent with the bioinformatics predications, and the corresponding amino acids coded by the missing exon 4 were amino acids $389-428$ of exostosin-1 protein, which form part of the conserved domain of exostosin (amino acids 110-396; Fig. 4).

Aberrantly reduced expression of EXT1/EXT2 genes. To investigate the potential effect of the splice mutation on the gene expression of EXT1/EXT2, the mRNA of EXT1/EXT2 and the abnormally spliced transcript of EXT1 gene was assessed in the patients with HME and normal controls. To distinguish the normal EXT1 transcript from the abnormally spliced transcript (with exon 4 skipping), the primers were designed with the downstream primer located in exon 4 of EXT1 for detecting the normal transcript, and the downstream primer spanned exons 3 and 5 of EXT1 to detect the abnormally spliced transcript. As depicted in Fig. 5, the levels of wild-type EXT1/EXT2 mRNA in patients with HME were significantly reduced, compared with the normal control $(\mathrm{P}<0.05)$. The level of the mutant EXT1 transcript was significantly increased in the proband, compared with the normal control and the mother $(\mathrm{P}<0.05)$.

Overexpression of EXT1-GFP fusion protein and aberrantly spliced RNA in vitro. Recombinant plasmids were successfully constructed and then packaged with lentivirus vectors, as confirmed by PCR and sequencing. After $48 \mathrm{~h}$ of infection with lentivirus, GV358-EXT1-FL and GV358-EXT1-DEL were overexpressed in the cells. RT-qPCR indicated that the expression levels of full-length transcript and aberrantly 
Table I. TA clone and sequencing results of the proband, mother of HME and normal control.

\begin{tabular}{lcccc}
\hline Subjects & $\begin{array}{c}\text { The clones with } \\
\text { skipped exon } 4\end{array}$ & $\begin{array}{c}\text { The clones without } \\
\text { skipped exon } 4\end{array}$ & $\begin{array}{c}\text { Total number } \\
\text { of clones }\end{array}$ & $\begin{array}{c}\text { Clones with skipped exon 4/ } \\
\text { total number of clones }\end{array}$ \\
\hline The proband $^{\mathrm{c}}$ & 26 & 13 & 39 & 0.667 \\
The mother & 24 & 17 & 41 & 0.585 \\
Normal control & 2 & 38 & 40 & 0.050 \\
\hline
\end{tabular}

${ }^{\mathrm{a} C}$ Compared with the proband, $\mathrm{P}=0.495\left(\chi^{2}\right.$ value, 0.564$)$; ${ }^{\mathrm{b}}$ compared with the normal control, $\mathrm{P}<0.001\left(\chi^{2}\right.$ value, 26.625); ${ }^{\mathrm{c}}$ compared with the normal control, $\mathrm{P}<0.001\left(\chi^{2}\right.$ value, 32.819).
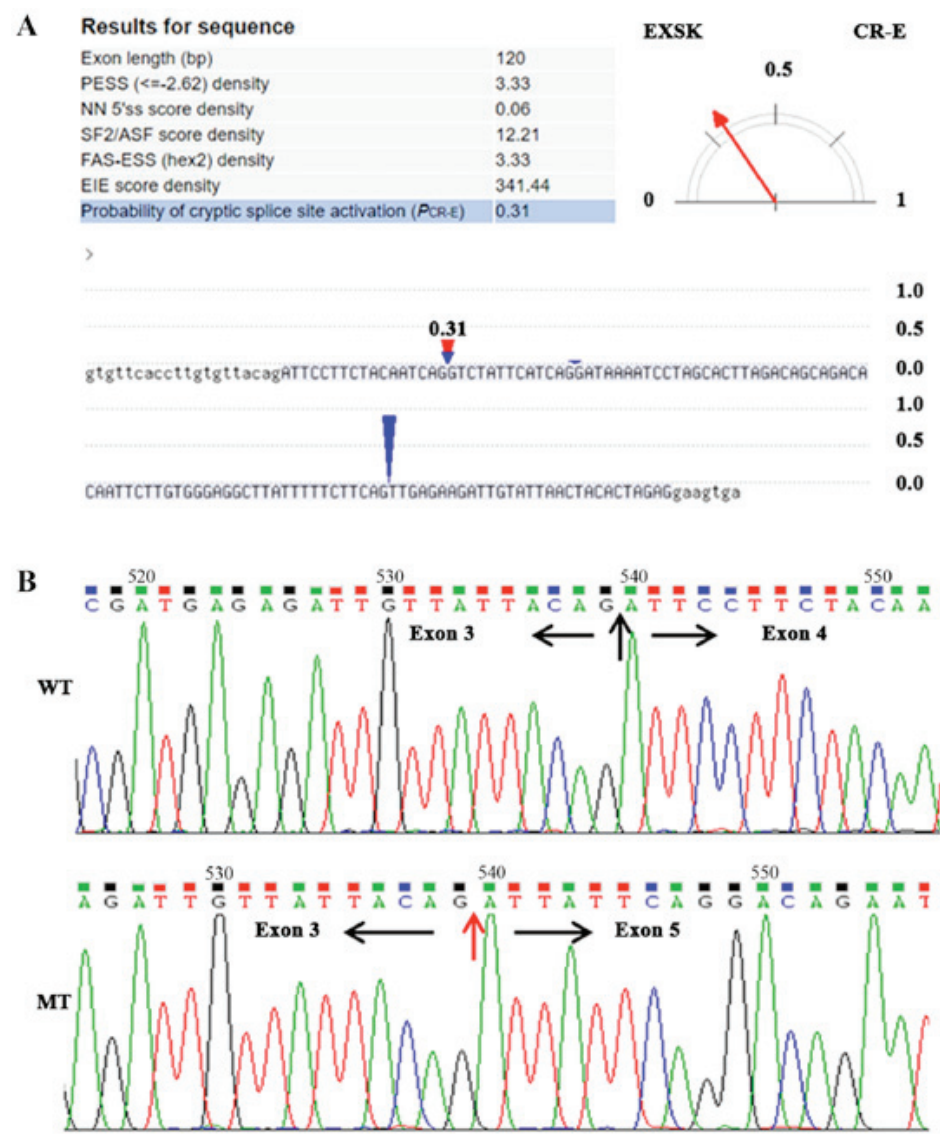

Figure 3. Bioinformatics prediction for c.1284+2del and TA clone-sequencing results of EXT1 mRNA. (A) Predictive output of c.1284+2del in EXT1 from CRYP-SKIP, the input EXT1 sequences involved exon 4 (capital letters) and adjacent intronic sequences (lower case letters). (B) TA cloning and sequencing results of the EXT1 gene. The arrows indicate the borders of adjacent exons. EXT1, exostosin glycosyltransferase 1; WT, wild type; MT, mutated type.

spliced transcript were significantly increased, compared with the empty vector infected with lentivirus (17.032- and 6.309-folds, respectively), and the level of aberrantly spliced transcript was significantly increased, compared with the full-length transcript (3.073-folds; $\mathrm{P}<0.05$; Fig. 6).

Increased expression of the truncated polypeptide, with no notable changes in subcellular localization. To investigate the cellular functionality of the aberrant polypeptide, and whether its expression level and subcellular location are different from EXT1-FL, western blot analysis was performed and the subcellular location was determined using laser scanning confocal microscope in cells expressing the lentiviral constructs. Western blotting revealed that the aberrant polypeptide was expressed by the vector, and at an increased level, compared with the wild-type protein (Fig. 7). However, the subcellular localization of the mutated polypeptide exhibited no alteration, compared with the full-length protein, with the majority of the EXT1-DEL and EXT1-FL protein located in the cytoplasm of Cos-7 cells (Fig. 8). However, endogenous EXT1 is generally considered to be located in the Golgi apparatus of the cytoplasm (30).

\section{Discussion}

The present study reported a novel heterozygous splice mutation (c.1284+2del) in intron 4 of the EXT1 gene identified in a three-generation family with HME. Mutation Taster and 

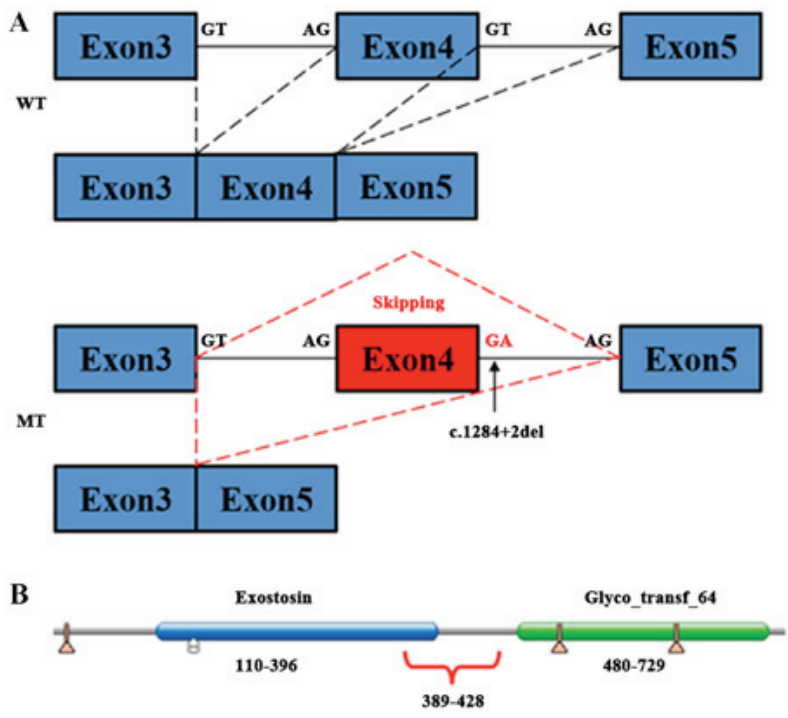

Figure 4. Splicing between adjacent exons and schematic drawing of exostosin-1. (A) Normal splicing between adjacent exons (WT), aberrant splicing and skipping of exon due to mutation in the important splicing site (MT). (B) Two important and conserved domains in exostosin-1. The blue column represents the domain of exostosin-1 that is coded by amino acids 110-396. The green column represents the domain of glycosyl transferase family 64 (Glyco_transf_64) coded by amino acids 480-729. The red bracket represents the coding area of skipped exon 4 (from 389 to 428 amino acids). WT, wild type; MT, mutated type.
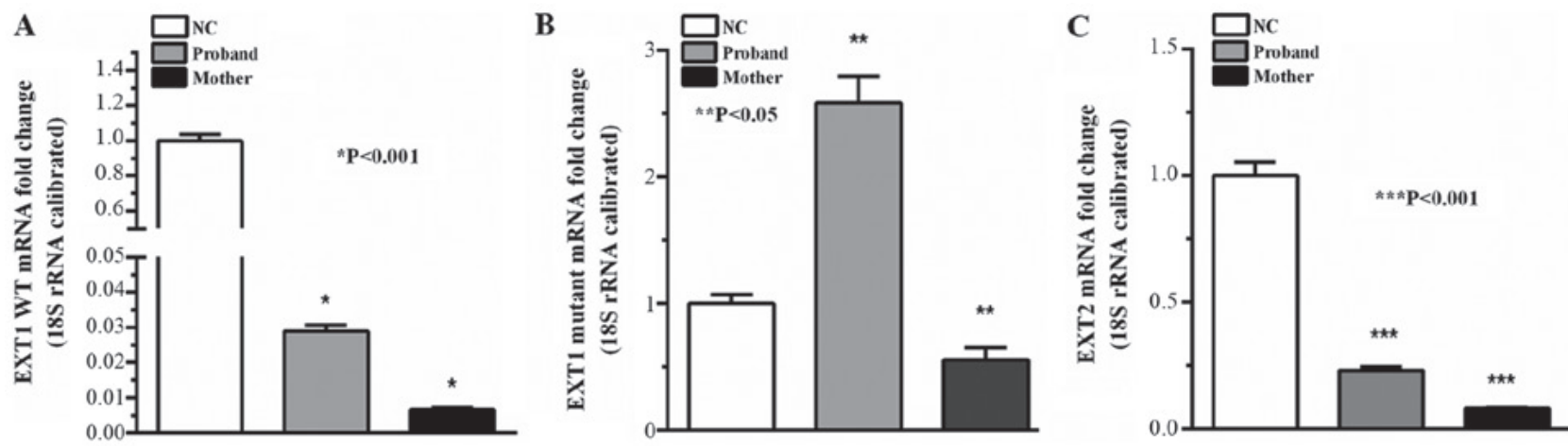

Figure 5. Reverse transcription-quantitative polymerase chain reaction analysis of the expression levels of EXT1/EXT2 mRNA and aberrantly spliced transcript of EXT1 gene in patients with hereditary multiple exostoses. (A) The relative levels of EXT1 wild-type mRNA were 0.0289/1.0 (the proband/NC) and 0.00654/1.0 (the mother/NC) $(\mathrm{P}<0.001)$. (B) The relative levels of EXT1 mutant mRNA were 2.58735/1.0 (the proband/NC) and 0.55260/1.0 (the mother/ NC) $(\mathrm{P}<0.05)$. (C) The relative levels of EXT2 mRNA were 0.23216/1.0 (the proband/NC) and 0.08038/1.0 (the mother/NC) $(\mathrm{P}<0.001)$. NC, normal control; EXT, exostosin glycosyltransferase.

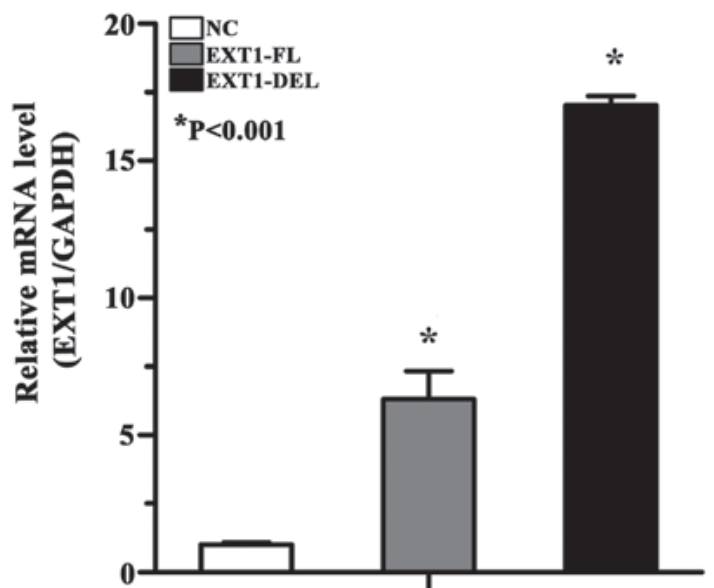

Figure 6. Reverse transcription-quantitative polymerase chain reaction analysis of the expression levels of EXT1 mRNA and aberrantly spliced transcript in 293-T cells infected with lentiviral vectors $(\mathrm{P}<0.001)$. NC, norma control; EXT1, exostosin glycosyltransferase-1; FL, full-length; DEL, aberrantly spliced transcript of EXT1.
PROVEAN predicted that c.1284+2del was a disease-causing mutation. The result of TA cloning and sequencing indicated that the mutation resulted in skipping of EXT1 exon 4 during mRNA splicing in the proband and his affected mother with no premature stop codon. RT-qPCR of the two patients revealed that expression levels of EXT1/EXT2 mRNA were reduced, compared with normal controls, and the levels of the abnormal EXT1 transcript (without exon 4) were increased in the proband, compared with his mother and normal control. Furthermore, the truncated peptide produced from the abnormally spliced transcript is potentially translated and expressed in cells without degradation via NMD. Additionally, the subcellular localization of the truncated peptide may be the same as protein produced from the wild-type EXT1 gene, and both proteins were observed to be localized to the cytoplasm in vitro.

Although the molecular mechanisms associated with HME are not fully understood, it is clear that HME is predominantly provoked by mutations in either/both of the EXT1 or EXT2 

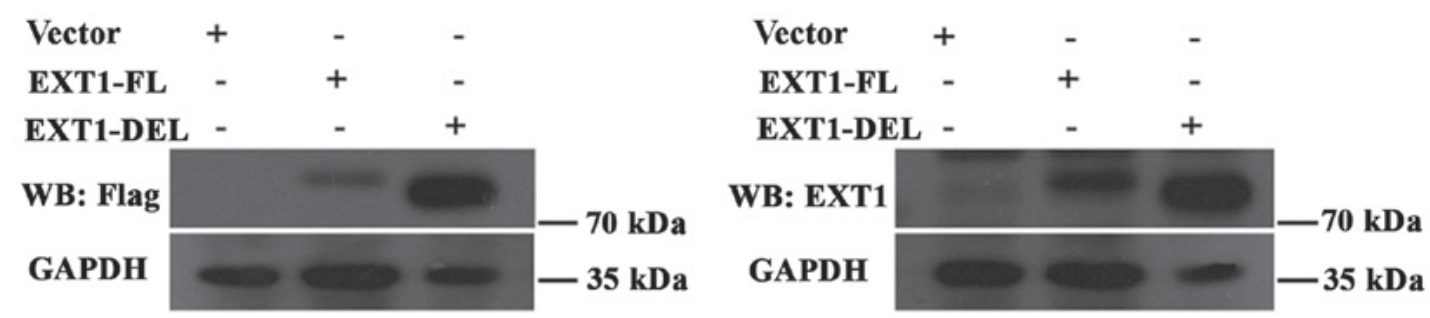

Figure 7. Western blot analysis of EXT1-FL and EXT1-DEL expression levels in 293-T cells infected with lentiviral vectors. Size of fusion protein of EXT1-FL, $84 \mathrm{kDa}$; size of fusion protein of EXT1-DEL, $80 \mathrm{kDa}$. Vector, empty vector lentivirus without targeted genes; EXT1, exostosin glycosyltransferase-1; FL, full-length; DEL, aberrantly spliced transcript of EXT1.
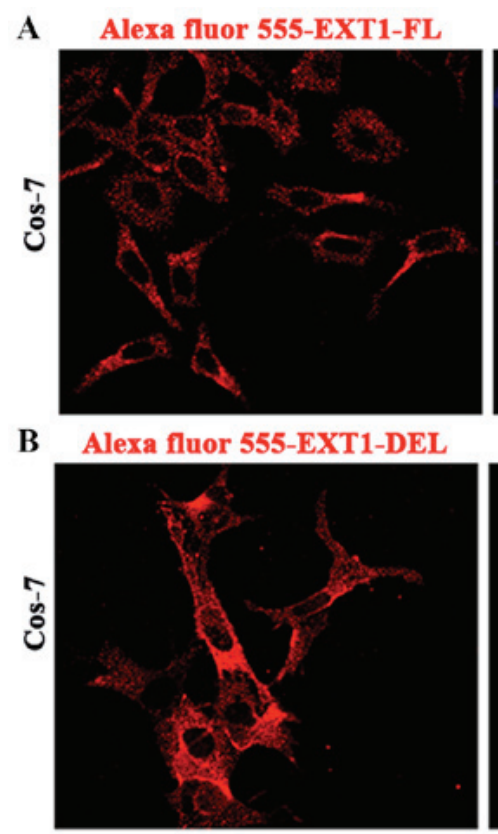

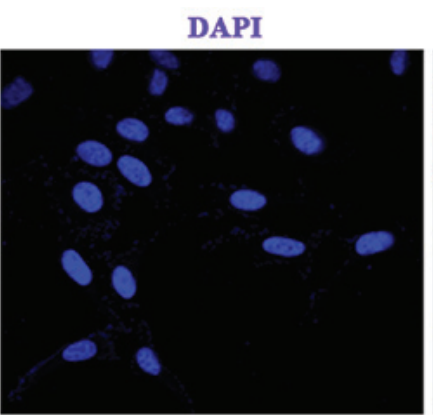

DAPI

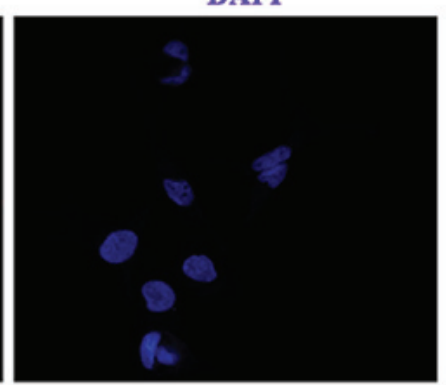

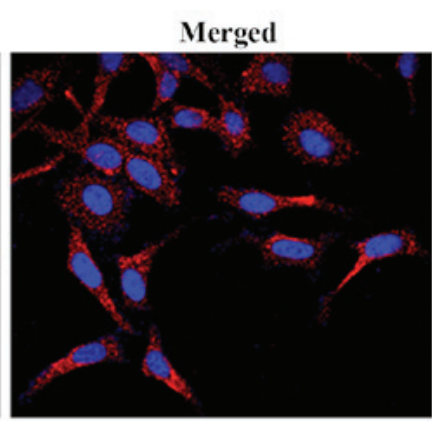

Merged

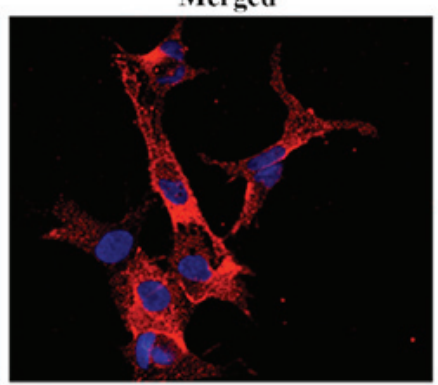

Figure 8. Immunofluorescence assay of expression and subcellular localization of (A) EXT1-FL and (B) EXT1-DEL in lentivirus-infected Cos-7 cells. EXT1, exostosin glycosyltransferase-1; FL, full-length; DEL, aberrantly spliced transcript of EXT1.

tumor suppressor genes. EXT1 accounts for 56-78\%, and EXT2 for 21-44\% of HME-causing mutations (11). Splicing mutations have been investigated to determine the molecular mechanism of HME $(22,23)$. Alternative splicing is one of the important mechanisms in regulating gene expression and protein diversity, splice sites ( $5^{\prime}$ and $3^{\prime}$ ), the branch site and the polypyrimidine sequence are the key splicing signals that have major roles in the splicing of pre-mRNA. If mutations occur in these sequences, the effective splicing of exons may be affected, which may interfere with subsequent transcription and translation $(20,21)$. The mutation identified in the present study was located at the 5' splice site of EXT1 exon 4, and was predicted and verified to cause exon 4 skipping. As the sequence length of exon 4 is $120 \mathrm{bp}$ and is a multiple of 3 , and the upstream coding region of exon 4 is also a multiple of 3 (1,164 bp), the skipping of exon 4 does not disturb the triplicate coding order of the downstream amino acids in EXT1 and premature stop code will not be created, which is different from other mutations reported in previous studies $(22,23)$.

TA cloning and sequencing results identified abnormal transcripts with skipping of exon 4 in the proband and his affected mother (66.7 and 58.5\%, respectively) at significantly increased levels, compared with the normal control participants $(\mathrm{P}<0.05)$; however, a few transcripts with exon 4 skipping were identified in the normal control (5\%; Table I), which may be explained by the phenomenon that alternative splicing frequently occurs in human genes with multiple exons (31). Furthermore, the number of abnormal transcripts in the proband was increased, compared with his mother, with no statistical significance between the two patients, potentially due to an insufficient number of clones detected. However, the results from RT-qPCR of EXT1/EXT2 mRNA and the abnormal spliced transcript of EXT1, which lacks exon 4 due to the splice mutation, in the two patients with HME were different from that of TA cloning and sequencing. The expression levels of wild-type EXT1/EXT2 mRNA in both patients were reduced, compared with the normal control (EXT1: proband, 0.02890; mother, 0.00654; and normal, 1.0; and EXT2: proband, 0.23216; mother, 0.08038; and normal, 1.0), particularly for EXT1 mRNA. By contrast, the level of the abnormal spliced EXT1 transcript with exon 4 skipping was significantly increased in the proband, compared with his mother and the normal control (proband, 2.58735; mother, 0.55260; and normal 1.0; $\mathrm{P}<0.05$ ). The difference between the TA cloning data and RT-qPCR analysis may be due to error in the TA cloning or the numbers of positive colonies analyzed 
may have been less than estimated. Additionally, although the levels of wild-type EXT1/EXT2 mRNA were also deceased in the mother, the level of the abnormally spliced EXT1 transcript with exon 4 skipping was significantly reduced, compared with the proband (Fig. 5; $\mathrm{P}<0.05$ ). This may be associated with the evidence that the clinical symptoms of male patients are prone to be more severe, compared with female patients, and it may also indicate that the severity increases with successive generations $(32,33)$.

Previous studies indicated that the mutated EXT1 and EXT2 were localized to the Golgi apparatus in vitro, which were similar to the wild-type genes $(30,34)$; however, the type of the mutations in these studies were truncated mutation (EXT2-Y419X) and missense mutations (EXT1-R340C and EXT2-D227N), and the produced protein was a truncated peptide that lacked the entire domain of Glyco_transf_64 (480-725aa) (EXT2-Y419X), or a single amino acid was changed (EXT1-R340C and EXT2-D227N), which were notably different from the identified splice mutation in the present study. As the splice mutation $(c .1284+2 \mathrm{del})$ in the present study just results in the deletion of partial amino acid sequences (389-428 aa), which is resident in the tail of the exostosin domain (110-396 aa) and the junction of the two domains of EXT1, whether the special peptide decays through NMD or not deserves intensive investigation. Additionally, if it is not decayed, where it anchors, and whether it is different from previous reports is also worth investigation.

Lentiviruses are effective and frequently-used tools that allow exogenous genes or exogenous short hairpin RNAs to be integrated into the host genome to achieve stable expression of the target sequence. 293-T cells and Cos-7 cells are common tool cells for efficiently expressing exogenous genes $(35,36)$. In the present study, a lentivirus was used to express the mutant EXT1 transcript lacking exon 4 in vitro. Overexpression of the abnormal transcript was confirmed by RT-qPCR in 293-T cells infected with the EXT1-DEL lentivirus; however, there were also small amounts of the abnormal transcript detected in cells infected with NC (empty vector) lentivirus (Fig. 6), indicating there may be some endogenous expression of this transcript in 293-T cells. Western blot analysis confirmed the expression of the truncated peptide. Other bands that were distinctly visible in the empty vector and EXT1-FL lanes were possibly caused by the relatively low specificity of the polyclonal EXT1 antibody used (Fig. 7B), while there was single band in either lane incubated with monoclonal anti-Flag antibody (Fig. 7A). In order to observe the subcellular localization of the abnormal peptide in the same host cells as a previous study (30), Cos-7 cells were also used as host cells and transfected with lentiviruses. Immunofluorescence demonstrated that the abnormal peptide was localized in the cytoplasm of Cos-7 cells, which was similar to the localization of EXT1-FL. Notably, the levels of the abnormal peptide were increased, compared with EXT1-FL (Fig. 8), which was consistent with the results of RT-qPCR and western blot analysis.

In conclusion, a novel splice mutation was identified in two patients from a family with HME in the present study. However, more family members did not enroll for co-segregation analysis of the mutation with disease status.
Expression levels of wild-type EXT1/EXT2 mRNA, which possess glycosyltransferase activity, were notably reduced due to the mutation in both patients, yet the level of an abnormally spliced transcript without the full functional domain was increased in the proband, compared with the normal control. The decrease in the copy numbers of the wild-type genes and increase in the abnormal transcript may be the pathogenic mechanism of HME in the family that participated in the present study. The abnormal transcript was detected in the patients with HME, and expression and localization of the protein product were assessed in vitro, revealing that the abnormal peptide was expressed and located in the cytoplasm of Cos-7 cells, which is in accordance with previous studies $(30,34)$. However, it was regrettable that there was no homologous structure in EXT1, except the C-terminal of Exostosin-1 (the domain of Glyco_transf_64), so computational biological analysis of the structure and function was lacking in the present study, in order to distinguish Exostosin-1 from the mutant protein; however, although the two proteins were detected in the cytoplasm of Cos-7 cells, it was not confirmed in vivo experiment. Furthermore, the observations in the present study was only derived from one cell line (Cos-7 cells). In conclusion, the biological function of wild-type EXT1/EXT2 proteins may not be affected by the emergence of increased levels of mutant EXT1/EXT2, but by the decrease in the levels of wild-type EXT1/EXT2 proteins, which will disrupt HS polymerization and chain elongation.

\section{Acknowledgements}

Not applicable.

\section{Funding}

This study was supported by grants from the Science and Technology Project of Fuzhou (grant no. 2015-S-141-14), Youth Research Project of Health and Family Planning in Fujian Province (grant no. 2016-2-41), Natural Science Funding Project in Fujian Province (grant no. 2016J01643) and the National Natural Science Foundation of China (grant no. 81772287).

\section{Availability of data and materials}

The datasets used and/or analyzed during the current study are available from the corresponding author on reasonable request.

\section{Authors' contributions}

XG designed the study concept and drafted the manuscript. ML performed data acquisition and analysis. ML, WC and GH revised the manuscript. WY was responsible for case collection and diagnosis. All authors reviewed the manuscript.

\section{Ethics approval and consent to participate}

Written informed consent was obtained from all participants, and the study was approved by the Ethics Committee of Fuzhou Second Hospital [Fuzhou, China; approval no. (2014) 63]. 


\section{Patient consent for publication}

The patients participated in the study agreed with the publication of the paper regarding their family research.

\section{Competing interests}

The authors declare that they have no competing interests.

\section{References}

1. Stieber JR and Dormans JP: Manifestations of hereditary multiple exostoses. J Am Acad Orthop Surg 13: 110-120, 2005.

2. Jones KB: Glycobiology and the growth plate: Current concepts in multiple hereditary exostoses. J Pediatr Orthop 31: 577-586, 2011.

3. Wicklund CL, Pauli RM, Johnston D and Hecht JT: Natural history study of hereditary multiple exostoses. Am J Med Genet 55: 43-46, 1995.

4. Schmale GA, Conrad EU III and Raskind WH: The natural history of hereditary multiple exostoses. J Bone Joint Surg Am 76: 986-992, 1994.

5. Porter DE, Lonie L, Fraser M, Dobson-Stone C, Porter JR, Monaco AP and Simpson AH: Severity of disease and risk of malignant change in hereditary multiple exostoses. A genotype-phenotype study. J Bone Joint Surg Br 86: 1041-1046, 2004.

6. Porter DE and Simpson AH: The neoplastic pathogenesis of solitary and multiple osteochondromas. J Pathol 188: 119-125, 1999.

7. Ahn J, Lüdecke HJ, Lindow S, Horton WA, Lee B, Wagner MJ, Horsthemke B and Wells DE: Cloning of the putative tumour suppressor gene for hereditary multiple exostoses (EXT1). Nat Genet 11: 137-143, 1995.

8. Hecht JT, Hogue D, Strong LC, Hansen MF, Blanton SH and Wagner M: Hereditary multiple exostosis and chondrosarcoma: Linkage to chromosome II and loss of heterozygosity for EXT-linked markers on chromosomes II and 8. Am J Hum Genet 56: 1125-1131, 1995.

9. Wuyts W and Van Hul W: Molecular basis of multiple exostoses: Mutations in the EXT1 and EXT2 genes. Hum Mutat 15: 220-227, 2000.

10. Szuhai K, Jennes I, de Jong D, Bovée JV, Wiweger M, Wuyts W and Hogendoorn PC: Tiling resolution array-CGH shows that somatic mosaic deletion of the EXT gene is causative in EXT gene mutation negative multiple osteochondromas patients. Hum Mutat 32: E2036-E2049, 2011

11. Jennes I, Pedrini E, Zuntini M, Mordenti M, Balkassmi S, Asteggiano CG, Casey B, Bakker B, Sangiorgi L and Wuyts W: Multiple osteochondromas: Mutation update and description of the multiple osteochondromas mutation database (MOdb). Hum Mutat 30: 1620-1627, 2009.

12. Stickens D, Clines G, Burbee D, Ramos P, Thomas S, Hogue D, Hecht JT, Lovett M and Evans GA: The EXT2 multiple exostoses gene defines a family of putative tumour suppressor genes. Nat Genet 14: 25-32, 1996.

13. McCormick C, Duncan G, Goutsos KT and Tufaro F: The putative tumor suppressors EXT1 and EXT2 form a stable complex that accumulates in the Golgi apparatus and catalyzes the synthesis of heparan sulfate. Proc Natl Acad Sci USA 97: 668-673, 2000.

14. Takei Y, Ozawa Y, Sato M, Watanabe A and Tabata T: Three Drosophila EXT genes shape morphogen gradients through synthesis of heparan sulfate proteoglycans. Development 131: 73-82, 2004.

15. Zak BM, Schuksz M, Koyama E, Mundy C, Wells DE, Yamaguchi Y, Pacifici M and Esko JD: Compound heterozygous loss of Ext1 and Ext2 is sufficient for formation of multiple exostoses in mouse ribs and long bones. Bone 48 : 979-987, 2011

16. Jones KB, Piombo V, Searby C, Kurriger G, Yang B, Grabellus F, Roughley PJ, Morcuende JA, Buckwalter JA, Capecchi MR, et al: A mouse model of osteochondromagenesis from clonal inactivation of Ext1 in chondrocytes. Proc Natl Acad Sci USA 107: 2054-2059, 2010.
17. Matsumoto K, Irie F, Mackem S and Yamaguchi Y: A mouse model of chondrocyte-specific somatic mutation reveals a role for Ext1 loss of heterozygosity in multiple hereditary exostoses. Proc Natl Acad Sci USA 107: 10932-10937, 2010.

18. Zuntini M, Pedrini E, Parra A, Sgariglia F, Gentile FV, Pandolfi M, Alberghini M and Sangiorgi L: Genetic models of osteochondroma onset and neoplastic progression: Evidence for mechanisms alternative to EXT genes inactivation. Oncogene 29: 3827-3834, 2010.

19. Reijnders CM, Waaijer CJ, Hamilton A, Buddingh EP, Dijkstra SP, Ham J, Bakker E, Szuhai K, Karperien M, Hogendoorn PC, et al: No haploinsufficiency but loss of heterozygosity for EXT in multiple osteochondromas. Am J Pathol 177: 1946-1957, 2010.

20. Barash Y, Calarco JA, Gao W, Pan Q, Wang X, Shai O, Blencowe BJ and Frey BJ: Deciphering the splicing code. Nature 465: 53-59, 2010 .

21. Wang GS and Cooper TA: Splicing in disease: Disruption of the splicing code and the decoding machinery. Nat Rev Genet 8: 749-761, 2007.

22. Tian C, Yan R, Wen S, Li X, Li T, Cai Z, Li X, Du H and Chen H: A splice mutation and mRNA decay of EXT2 provoke hereditary multiple exostoses. PLoS One 9: e94848, 2014.

23. Chen XJ, Zhang H, Tan ZP, Hu W and Yang YF: Novel mutation of EXT2 identified in a large family with multiple osteochondromas. Mol Med Rep 14: 4687-4691, 2016.

24. Bovée JV: Multiple osteochondromas. Orphanet J Rare Dis 3 3, 2008

25. Wuyts W, Radersma R, Storm K and Vits L: An optimized DHPLC protocol for molecular testing of the EXT1 and EXT2 genes in hereditary multiple osteochondromas. Clin Genet 68: 542-547, 2005.

26. Divina P, Kvitkovicova A, Buratti E and Vorechovsky I: Ab initio prediction of mutation-induced cryptic splice-site activation and exon skipping. Eur J Hum Genet 17: 759-765, 2009.

27. Desmet FO, Hamroun D, Lalande M, Collod-Béroud G, Claustres M and Béroud C: Human Splicing Finder: An online bioinformatics tool to predict splicing signals. Nucleic Acids Res 37: e67, 2009.

28. Livak KJ and Schmittgen TD: Analysis of relative gene expression data using real-time quantitative PCR and the 2(-Delta Delta C(T)) method. Methods 25: 402-408, 2001.

29. Lizée G, Aerts JL, Gonzales MI, Chinnasamy N, Morgan RA and Topalian SL: Real-time quantitative reverse transcriptase-polymerase chain reaction as a method for determining lentiviral vector titers and measuring transgene expression. Hum Gene Ther 14: 497-507, 2003.

30. Kobayashi S, Morimoto K, Shimizu T, Takahashi M, Kurosawa H and Shirasawa T: Association of EXT1 and EXT2, hereditary multiple exostoses gene products, in Golgi apparatus. Biochem Biophys Res Commun 268: 860-867, 2000.

31. Xie BB, Li D, Shi WL, Qin QL, Wang XW, Rong JC, Sun CY, Huang F, Zhang XY, Dong XW, et al: Deep RNA sequencing reveals a high frequency of alternative splicing events in the fungus Trichoderma longibrachiatum. BMC Genomics 16: 54, 2015.

32. Clement ND and Porter DE: Hereditary multiple exostoses: Anatomical distribution and burden of exostoses is dependent upon genotype and gender. Scott Med J 59: 35-44, 2014.

33. Pedrini E, Jennes I, Tremosini M, Milanesi A, Mordenti M, Parra A, Sgariglia F, Zuntini M, Campanacci L, Fabbri N, et al: Genotype-phenotype correlation study in 529 patients with multiple hereditary exostoses: Identification of 'protective' and 'risk' factors. J Bone Joint Surg Am 93: 2294-2302, 2011.

34. Busse M, Feta A, Presto J, Wilén M, Grønning M, Kjellén L and Kusche-Gullberg M: Contribution of EXT1, EXT2, and EXTL3 to heparan sulfate chain elongation. J Biol Chem 282: 32802-32810, 2007.

35. Bobadilla S, Sunseri N and Landau NR: Efficient transduction of myeloid cells by an HIV-1-derived lentiviral vector that packages the Vpx accessory protein. Gene Ther 20: 514-520, 2013.

36. Nukuzuma S, Nakamichi K, Kameoka M, Sugiura S, Nukuzuma C, Miyoshi I and Takegami T: Efficient propagation of progressive multifocal leukoencephalopathy-type JC virus in COS-7-derived cell lines stably expressing Tat protein of human immunodeficiency virus type 1. Microbiol Immunol 54: 758-762, 2010.

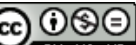

This work is licensed under a Creative Commons Attribution-NonCommercial-NoDerivatives 4.0 International (CC BY-NC-ND 4.0) License. 